\title{
UMA CONSTRUÇÃO HISTÓRICA DAS TÉCNICAS DA TRANSFORMADA INTEGRAL CLÁSSICA (CITT) E GENERALIZADA (GITT): ASPECTOS INICIAIS
}

\author{
A HISTORICAL CONSTRUCTION OF CLASSICAL INTEGRAL \\ TRANSFORM TECHNIQUE (CITT) AND GENERALIZED INTEGRAL \\ TRANSFORM TECHNIQUE (GITT): INITIAL ASPECTS
}

\author{
Reynaldo D'Alessandro Neto ${ }^{1}$ \\ IGCE - UNESP - Rio Claro
}

\begin{abstract}
Resumo
Estes resultados parciais de uma pesquisa de doutorado do Programa de Pós-Graduação em Educação Matemática da UNESP - Rio Claro, insere-se na linha de pesquisa Relações entre História e Educação Matemática e tem como objetivo descrever a evolução histórica que culmina na concepção da Técnica da Transformada Integral Clássica, e as motivações que levaram a sistematização do seu modelo generalizado. As técnicas têm como foco resolver Equações Diferenciais Parciais (EDP) a princípio não tratáveis pelas teorias clássicas, como o conhecido método da separação de variáveis. Pretendemos fazer uma construção histórica, considerando o contexto do seu surgimento e desenvolvimento, passando pelas diversas modificações ao longo dos estudos e necessidades de se tornar uma técnica mais competitiva para a evolução do mundo tecnológico. Para atingir esse objetivo, faremos uma abordagem historiográfica que começa ao descrevermos algumas motivações históricas dos desenvolvimentos da Transformada Integral, e as principais ideias da Transformada Integral Finita por N.S. Koshlyakov. Além dos estudos detalhados realizados por G.A. Grinberg (1948), que generaliza os métodos de Koshlyakov, para o caso de mudança das propriedades do meio na direção da coordenada ao longo da qual a transformação é executada. E a aplicação de M.D. Mikhailov (1972), que propõe um núcleo de núcleo de processamento geral que unificou as várias transformações desenvolvidas até então, obtendo a solução para a equação da difusão linear em regiões finitas. Para assim, podermos entender esses movimentos que são precursores da proposta da Técnica da Transformada Integral Clássica (CITT - Classical Integral Transform Technique), de Özisik e Murray (1974). E, por fim, dos conceitos que surgiram com o formalismo da Técnica Transformada Integral Generalizada (GITT Generalized Integral Transform Technique), proposta por Özisik e Mikhailov (1984). Nesses resultados parciais de pesquisa, apresentamos os passos descritos acima até a contribuição de Mikhailov (1972), que serão finalizados com a análise dos escritos que fundamentam a CITT e GITT.
\end{abstract}

\footnotetext{
1 reynaldo.dalessandro@gmail.com.
} 
Palavras-chave: História da Matemática; Equação Diferencial Parcial - EDP; Transformada Integral; Técnica da Transformada Integral Clássica - (CITT); Técnica da Transformada Integral Generalizada - (GITT).

\begin{abstract}
These partial results of $\mathrm{PhD}$ research of the Program in Mathematical Education of UNESP - Rio Claro, is part of the research line Relations between History and Mathematical Education and aims to describe the historical evolution that culminates in the conception of the Technique. Classical Integral Transform, and the motivations that led to the systematization of its generalized model. The techniques focus on solving partial differential equations (EDP) at first not treatable by classical theories, such as the well-known method of variable separation. We intend to make a historical construction, considering the context of its emergence and development, going through the various modifications throughout the studies and the need to become a more competitive technique for the evolution of the technological world. To achieve this goal, we will take a historiographical approach that begins by describing some historical motivations of developments in the Integral Transform, and the main ideas of the Finite Integral Transform by N.S. Koshlyakov. In addition to detailed studies by G.A. Grinberg (1948), who generalizes Koshlyakov's methods, in the case of changing the properties of the medium in the direction of the coordinate along which the transformation is performed. And the application of M.D. Mikhailov (1972), who proposes a general processing nucleus that unified the various transformations developed so far, obtaining the solution for the finite region linear diffusion equation. Thus, we can understand these movements that are precursors of Özisik and Murray's (1974) proposal of the Classical Integral Transform Technique (CITT). And finally, the concepts that emerged with the formalism of the Generalized Integral Transform Technique (GITT), proposed by Özisik and Mikhailov (1984). In these partial research results, we present the steps described above until the contribution of Mikhailov (1972), which will be finalized with the analysis of the writings that underlie the CITT and GITT.
\end{abstract}

Keywords: Math History; Partial Differential Equations - PDE; Integral Transform; Technique of the Classical Integral Transform - (CITT); Generalized Integral Transformation Technique - (GITT).

\title{
Introdução
}

Durante o mestrado, participamos do grupo de Simulação e Experimentos para Microfiltração e Ultrafiltração da Universidade Federal de São Carlos - Campus Sorocaba. Nesse grupo, estudávamos modelagens matemática relacionadas aos problemas de filtração tangencial, dentre as soluções que se aplicavam aos problemas, as Técnicas da Transformada Integral Clássica (CITT) e Generalizada (GITT) eram as mais estudadas. E com o interesse sobre o assunto, tivemos um contato direto com diversos materiais de estudo que trazem embasamento relacionado a suas aplicações. 
E durante o processo da escrita da dissertação, percebemos a necessidade de um estudo histórico sobre a teoria, devido a dificuldade de se encontrar informações relativas ao tema. Assim, iniciamos um levantamento e verificamos a originalidade de tal estudo.

E sabemos da importância de se fazer uma pesquisa histórica de conteúdos matemáticos, pois, através delas podemos perceber que todas as descobertas se deram com o estudo de outras feitas por matemáticos que viveram anteriormente, como se cada nova descoberta fosse apenas mais uma etapa da construção de um conhecimento universal na busca de resolver algum problema.

Com isso, uma pesquisa que envolva a história de um conteúdo matemático tem grande relevância para a Matemática, pois pode servir como um instrumento para o futuro pesquisador como um compilado de informações relevantes, no nosso caso, da técnica estudada.

E assim, podemos entender quais foram os problemas de aplicação que motivaram o desenvolvimento de tal conteúdo. Como comenta D’Ambrosio (2000, p.162):

Em todas as conceituações, os estudos de História dependem
fundamentalmente do reconhecimento de fatos, de datas e de nomes e de
interpretação ligados ao objeto de nosso interesse, isto é, do corpo de
conhecimentos em questão. Esse reconhecimento depende de uma definição
do objeto de nosso interesse. No nosso caso específico, depende do que se
entende por Matemática.

No nosso caso, estamos especialmente interessados no desenvolvimento de métodos de soluções para Equações Diferenciais Parciais (EDP). Sabemos que diversas técnicas na forma de métodos puramente numéricos, métodos analíticos e ainda métodos híbridos analítico-numéricos foram desenvolvidos.

Dentre as técnicas analíticas, temos o clássico método da Separação de Variáveis, importante técnica que determina a solução de equações diferenciais parciais, mas de abrangência bastante reduzida, uma vez que o modelo matemático deve obedecer a uma série de restrições para que o problema seja separável.

Para resolver EDP a princípio não tratáveis pela Separação de Variáveis, surgem novas abordagens, dentre essas, a Técnica da Transformada da Integral Clássica (CITT). O método elimina totalmente a necessidade de o problema ser separável, por exemplo, as equações diferenciais que governam os processos de difusão (principalmente a de calor).

Segundo o professor Renato Machado Cotta, no seu artigo de 2012, intitulado The Unified Integral Transforms (UNIT) algorithjm with total and partial transformation: A 
Uma Construção Histórica das Técnicas da Transformada Integral Clássica (CITT) e Generalizada (GITT): Aspectos Iniciais

Reynaldo D'Alessandro Neto

tribute to Prof. Mikhail D. Mikhailov, e o professor Aleksei Luikov, no seu livro Heat and Mass Transfer de 1972, as ideias precursoras que culminaram na concepção da CITT foram sugeridas por N.S. Koshlyakov em seu livro Basic Differential Equations of Mathematical Physics, de 1936.

Nesse livro, Koshlyakov define um tipo de transformação integral que ficou conhecida posteriormente como Transformada Integral Finita.

Depois das primeiras ideias, G.A. Grinberg fez um desenvolvimento mais detalhado das transformações integrais no livro Selected Problems of Mathematical Theory of Electrical and Magnetic Effects, de 1948.

Mas foi o artigo escrito por M.D. Mikhailov em 1972 intitulado General Solution of the Heat Equation in Finite Regions, que deu uma contribuição extremamente significativa para a consolidação da técnica de Koshlyakov. No estudo, o autor elabora uma proposta de um núcleo de processamento geral que unificou as várias transformações ao obter uma solução geral para a equação da difusão linear em regiões finitas.

Finalmente, M.N. Özisik e R.L. Murray em 1974 no artigo On the Solution of Linear Diffusion Problems With Variable Boundary Condition Parameters, utilizaram as ideias dos autores citados anteriormente e, aplicaram pela primeira vez uma nova técnica para a resolução de sistemas de Equações Diferenciais Parciais (EDP), chamada posteriormente de Técnica da Transformada Integral Clássica (CITT - Classical Integral Transform Technique).

Em alguns casos de problemas propostos por Özisik e Murray (1974) se caracterizava pela presença de termos não transformáveis pela CITT, que mesmo assim foram inseridos na fórmula de inversão. Para a recuperação do problema original, foram obtidas soluções aproximadas deste sistema de equações diferenciais, nasce aí a natureza híbrida analítico-numérica do procedimento adotado e os primeiros passos na Técnica Transformada Integral Generalizada (GITT - Generalized Integral Transform Technique).

Em 1984, Özisik e Mikhailov, no livro Unified Analysys and Solution of Heat and Mass Difusion, apresentaram uma maneira sistemática de aplicação da Técnica da Transformada Integral para a solução de diversos problemas lineares de difusão, divididos em sete grandes classes que foram definidas partindo de inúmeros problemas de 
Uma Construção Histórica das Técnicas da Transformada Integral Clássica (CITT) e Generalizada (GITT): Aspectos Iniciais

Reynaldo D'Alessandro Neto

transferência de calor e massa disponíveis na literatura. Desse trabalho surgiram os formalismos da GITT.

Essas evoluções, segundo Cotta (2012), ocorreram devido ao período da corrida tecnológica espacial, onde diversos países do Leste Europeu como a URSS e Bulgária, tiveram suas pesquisas concentradas no desenvolvimento de ferramentas analíticas, tal como a Técnica da Transformada Integral, buscando economizar os recursos computacionais quase que indisponíveis nestes países.

Concomitantemente, os Estados Unidos e Europa concentravam-se no desenvolvimento aos métodos puramente numéricos, como os conhecidos: Métodos de Diferenças Finitas, Elementos Finitos e Volumes Finitos, que se tornaram viáveis com o advento do computador.

E assim, durante a década de 1980, vários pesquisadores norte-americanos, soviéticos e búlgaros trabalharam conjuntamente, visando desenvolver técnicas híbridas analítico-numéricas, que conseguissem unir as características positivas de cada tipo de abordagem do problema.

Como essa introdução ao tema, podemos delimitar nossos objetivos, que para esta pesquisa será descrever a evolução histórica que culmina na concepção da Técnica da Transformada Integral Clássica (CITT) e do seu modelo generalizado (GITT). Com a intenção de formar um compilado para futuros pesquisadores e professores que pesquisem e/ou ensinem na área.

Como iremos apresentar um resultado parcial da nossa pesquisa, este texto conterá o que foi melhor desenvolvido na pesquisa até o presente momento. Com isso, traremos os passos descritos acima até a contribuição de Mikhailov (1972).

\section{Motivações históricas e o desenvolvimento de Koshlyakov}

Segundo Deakin (1985) no artigo Euler's Invention of Integral Transform, os estudos considerados como precursores das Transformações Integrais são do matemático Leonhard Euler. Deakin comenta que as primeiras ideias de transformação devem ser encontradas em um fragmento de Euler (1763) dedicado a resolução de uma equação diferencial em específico. E, mais tarde, em um capítulo do conhecido livro Institutiones Calculi Integralis de Euler (1769), onde o tratamento é mais completo e geral, mesmo sendo muito incipiente. Esses trabalhos envolvem transformações integrais de grande reconhecimento.

Número Especial - IV Seminário Cearense de História da Matemática

Boletim Cearense de Educação e História da Matemática - Volume 07, Número 20, 80 - 92(2020)

DOI: 10.30938/bocehm.v7i20.2613 
Cotta et al. (2017) no texto Analytical Methods in Heat Transfer, comentam que no trabalho de Fourier de 1807, Théorie de la Propagation de la Chaleur dans les Solides, e seu tratado, Theorie Analytique de la Chaleur de 1822, foi consolidada a formulação matemática de fenômenos de condução de calor em termos de uma equação diferencial parcial para a temperatura dentro de um corpo, com as variáveis de espaço e tempo.

De acordo com Cotta et al. (2017), somente mais tarde que Dirichlet foi capaz de fornecer uma solução exata para cada formulação diferencial parcial, incluindo análise da equação de condução de calor no formato retangular, sistemas de coordenadas cilíndricas e esféricas.

E assim, algumas soluções das EDO precisavam satisfazer a propriedade da ortogonalidade, e por isso geram infinitas soluções em forma de expansões e séries infinitas de funções onde o produto entre elas é zero (condição de ortogonalidade). Foi aí que a teoria desenvolvida pelos dois matemáticos franceses entre 1829 e 1837, SturmLiouville, obteve uma grande aplicação.

Desse modo, foi aberto um novo caminho para uma metodologia de solução analítica mais abrangente, que ficou conhecida como o método da Transformada Integral. Essa técnica de resolução possui diversas abordagens desenvolvidas ao longo da história, dentre as mais conhecidas estão a Transformada de Laplace e de Fourier.

Renato Machado Cotta, no seu artigo de 2012, intitulado The Unified Integral Transforms (UNIT) algorithjm with total and partial transformation: A tribute to Prof. Mikhail D. Mikhailov, nos apresenta diversos dados históricos que estão diretamente relacionados as propostas de M.D. Mikhailov, M.N. Özisik, e, posteriormente, na concepção de um algoritmo que facilita a implementação da GITT.

Nesse caminho, Cotta (2012) explica que o Trabalho de N.S. Koshlyakov (1936) nos forneceu uma primeira ideia ao lidar com equações de difusão não homogêneas e condições de contorno pelo método das Transformações Integrais Finitas.

No livro de 1936, intitulado Basic Differential Equations of Mathematical Physics, do russo, Osnovnye Differentsial'nye Uravneniya Matematicheskoi Fiziki, N.S. Koshlyakov, estuda uma série de problemas físicos que envolvem EDP.

Com o auxílio do Prof. Renato Cotta, encontramos o exemplar original em Russo de 1936 e comparamos com a publicação de 1964 intitulada Differential Equations of Mathematical Physics, escrita por N.S. Koshlyakov, M.M. Smirnov e E.B. Gliner. Com 
Uma Construção Histórica das Técnicas da Transformada Integral Clássica (CITT) e Generalizada (GITT): Aspectos Iniciais

Reynaldo D'Alessandro Neto

a análise das duas obras, verificamos que a presença da Transformada na publicação de 1936 está de forma diluída no texto, durante a solução de cada problema proposto pelo autor. Já no livro de 1964, vemos um capítulo inteiro dedicado ao trato da Transformada Integral, de um modo didático e compreensível. Assim, escolhemos a obra de 1964 para a realização do nosso estudo.

O objeto de nosso estudo está presente na parte IV desse livro. No primeiro capítulo dessa seção e XXXI do livro, vemos o título: The use of integral operators in solving problems in mathematical physics.

\section{Part IV. Supplementary material}

\section{Chapter XXXI. The use of integral operators in solving problems in} mathematical physics

1. Basic definitions. Method of application of integral operators 522

2. Conditions allowing the use of integral operators $\mathbf{5 2 2}$

3. Finite integral transfor mations $\quad \mathbf{5 2 5}$

4. Integral transformations in infinite intervals $\quad 530$

5. Summary of the results $\quad 537$

Figura 1 - Sumário do Capítulo XXXI

No início do item 1 desse capítulo, Koshlyakov define "operador integral" e explica que esses termos são aplicados aos resultados de uma transformação. Assim, para Koshlyakov, a transformação da forma:

$$
\begin{gathered}
\bar{u}\left(\gamma_{1}, \gamma_{2}, \gamma_{3}, \ldots, \gamma_{m} ; x_{m+1}, \ldots, x_{n}\right) \\
=\iint_{S} K\left(x_{1}, \ldots, x_{m} ; \gamma_{1}, \gamma_{2}, \ldots, \gamma_{m}\right) \mathrm{u}\left(x_{1}, \ldots x_{n}\right) d x_{1 . .} d x_{m}
\end{gathered}
$$

É a Operação (Transformada) Integral na região $S$ pela qual a função original $u\left(x_{1}, x_{2}, \ldots, x_{n}\right)$ em relação às $n$ variáveis é transformada em uma função $\bar{u}\left(\gamma_{1}, \gamma_{2}, \gamma_{3}, \ldots, \gamma_{m} ; x_{m+1}, \ldots, x_{n}\right)$ com $m$ variáveis $\left(\gamma_{1}, \gamma_{2}, \ldots . \gamma_{m}\right)$ e $n-m$ variáveis $\left(x_{m+1}, \ldots, x_{n}\right)$, com núcleo (Kernel) $K\left(x_{1}, x_{2}, \ldots, x_{m} ; y_{1}, y_{2}, \ldots, y_{m}\right)$ dado que $m \leq n$.

Koshlyakov, após demonstrar nos itens do seu capítulo, as definições básicas, condições de utilização e o desenvolvimento da técnica para intervalos finitos e infinitos, finaliza o capítulo, no item 5, com um resumo para facilitar o uso dos métodos dos operadores integrais. Dentre as principais relações e passos, destacamos o núcleo (Kernel) 
do operador proposto por Koshlyakov, que se tornou muito aplicável nas resoluções de EDP que possuem correlação com fenômenos físicos:

$$
K\left(x_{i}, \gamma\right)=\frac{1}{C_{\gamma}} \rho\left(x_{i}\right) \bar{K}_{\gamma}\left(x_{i}\right)
$$

Onde $C_{\gamma} ; \rho\left(x_{i}\right) ; \bar{K}\left(x_{i}, \gamma\right)$ são funções determinadas por Koshlyakov, utilizando os coeficientes da EDP dada. E, a solução problema original, expressa em termos da solução $\bar{u}_{\gamma}$ do problema transformado, por meio das séries:

$$
u=\sum_{\gamma=1}^{\infty} \bar{u}(\gamma) \bar{K}_{\gamma}\left(x_{i}\right)
$$

Por fim, Koshlyakov lista as várias operações que devem ser realizadas no caso de uma Transformada Integral dentro de limites finitos, na seguinte ordem:

(1) Estabelecer a validade de usar um operador no problema em questão.

(2) Calcule (a partir dos valores dos coeficientes da expressão diferencial) as funções $C_{\gamma} ; \rho\left(x_{i}\right) ; \bar{K}\left(x_{i}, \gamma\right)$.

(3) Encontre a função $K\left(x_{i}, \gamma\right)$.

(4) Encontre o kernel do operador direto.

(5) Use as relações para escrever o problema transformado.

(6) Encontre a solução do problema transformado. (Aqui, em particular, Koshlyakov sugere a aplicação repetida de transformações integrais).

(7) Escreva a solução do problema original na forma da série.

Destacamos aqui, seu método para intervalos finitos, porém, o autor também apresenta de maneira análoga sua metodologia para intervalos infinitos.

Com toda a descrição acima, vemos a contribuição de Koshlyakov para a construção inicial dessa Transformada Integral, ao mostrar o uso dos operadores integrais para intervalos finitos e infinitos, com núcleos (kernel) e operadores inversos definidos, bem como toda metodologia para se encontrar cada coeficiente ou função necessária para a técnica. É interessante ressaltar que o método de Koshlyakov é chamado em diversos livros, como nos de Luikov (1972) e Cotta (2012), de Transformada Integral Finita.

Acreditamos que isso se deve ao fato de que a técnica para intervalos finitos se tornou a mais utilizada em problemas de valor de contorno, ou seja, equações diferenciais parciais que possuem condições iniciais para sua resolução. Além de que, após a análise e leitura da proposta de Koshlyakov para intervalos infinitos, vemos que o autor faz uso 
Uma Construção Histórica das Técnicas da Transformada Integral Clássica (CITT) e Generalizada (GITT): Aspectos Iniciais

Reynaldo D'Alessandro Neto

de manipulações matemáticas e sugere a utilização de transformadas conhecidas, como as de Laplace, Fourier e Hankel. Deixando para alguns casos especiais a utilização do seu método proposto para intervalos infinitos.

\section{Desenvolvimento das Transformações Integrais de G.A. Grinberg (1948)}

Por não encontrar a literatura original de G.A. Grinberg - Selected Problems of Mathematical Theory of Electrical and Magnetic Effects de 1948, foi pesquisado livros que fizessem menções e discussões sobre sua contribuição para o desenvolvimento da Transformada Integral. Dentre os estudos encontrados, foi o de A.V. Luikov (1972) que mais detalhou o desenvolvimento realizado por Grinberg.

Para Luikov (1972), as limitações dos métodos da Transformada de Fourier, Hankel e de Laplace, levou à criação de métodos da Transformada Integral Finita. E, mesmo para problemas que podem ser resolvidos pelos métodos clássicos com a ajuda da série de Fourier ou Fourier-Bessel, a Transformada Integral Finita é um método que pode ser preferível do ponto de vista da simplicidade de abordagem.

Segundo Luikov (1972), após a proposta de Koshlyakov, a teoria foi melhor desenvolvida por Grinberg (1948), que generalizou os métodos para o caso de mudança de propriedades do meio na direção dessa coordenada ao longo da qual a transformação é executada, principalmente em problemas de cunho físico (eletricidade e magnetismo)

Nesse desenvolvimento detalhado, os kernels $K(p, x)$ utilizados são as transformações integrais finitas de Fourier e Hankel, escolhidos apropriadamente e com solução encontrada a partir do problema de Sturm-Liouville. Se as autofunções desse problema forem designadas por $X_{n}(x)$ e a ponderação das funções $\rho(x)$ dentro do intervalo $[a, b]$, então temos:

$$
K(p, x)=\rho(x) X_{n}(x)
$$

Dessa forma, Grinberg faz a sugestão do núcleo e o define a partir dos limites de integração. Para Luikov, Grinberg (1948) desenvolve um método que, além de facilitar a escolha do kernel, tem como ideia principal a escolha do mesmo em conformidade com a equação diferencial e as condições de contorno, isto é, levando em consideração forma geométrica do corpo e da lei de sua interação com o meio físico em questão. 
Uma Construção Histórica das Técnicas da Transformada Integral Clássica (CITT) e Generalizada (GITT): Aspectos Iniciais

Reynaldo D'Alessandro Neto

\section{Desenvolvimento das Transformações Integrais de M.D. Mikhailov (1972)}

Após o desenvolvimento de Grinberg (1948), os conceitos da Técnica da Transformada Integral Finita já estavam bem documentados em trabalhos científicos como nos livros: Integral Transform in Mathematical Physics de C.J. Tranter (1962) e The Use of Integral Transforms de I.M. Sneddon (1972). Mas foi a obra intitulada: General Solution of the Heat Equation in Finite Regions, publicada como artigo em 1972, por M.D. Mikhailov no International Journal Engineering Sciences, que deu uma contribuição extremamente significativa para consolidação a técnica.

Segundo Cotta (2012), a concepção da GITT sofreu uma grande influência das publicações de M.D. Mikhailov, em um período extremamente produtivo. Dentre essas produções, inclui-se o seu trabalho de 1972.

Mikhailov, no seu trabalho, propõe um núcleo de núcleo de processamento geral que unificou as várias transformações desenvolvidas até então, obtendo a solução geral para a equação da difusão linear em regiões finitas.

O objetivo do estudo de Milhailov será solucionar um problema de valor de contorno, ou seja, resolver a Equação Diferencial Parcial:

$$
\begin{aligned}
\varphi(\tau) w(M) \frac{\partial T(M, \tau)}{\partial \tau} & \\
& =\operatorname{div}[(K(M) \operatorname{grad} T(M, \tau)]+[\beta(\tau) w(M)-\rho(M)] T(M, \tau) \\
& +P(M, \tau)
\end{aligned}
$$

Onde div é divergente e grad o gradiente no espaço $M$. Com condição inicial:

$$
T(M, 0)=f_{0}(M)
$$

E condição de contorno geral:

$$
A(N) \frac{\partial T(N, \tau)}{\partial n}+B(N) T(N, \tau)=f(N, \tau)
$$

Para resolver a equação nas condições dadas, segue a proposta de Transformada Integral Finita de Mikhailov:

$$
\tilde{T}_{i}(\tau)=\int_{V} w(M) \psi_{i}(M) T(M, \tau) d V
$$

Que segundo o autor será utilizada. É interessante reparar que a transformada sugerida possui um núcleo muito parecido com a sugestão de Grinberg (1948), caracterizado pela inserção da função peso $w(M)$ e as autofunções $\psi_{i}(M)$. Seguindo, a 
partir da ortogonalidade das autofunções $\psi_{i}(M)$, que $T(M, \tau)$ pode ser expandido na série (Operador Inverso) abaixo:

$$
T(M, \tau)=\sum_{i=1}^{\infty} \tilde{T}_{i}(\tau)=\int_{V} G_{i} \psi_{i}(M) \tilde{T}_{i}(\tau)
$$

Seguindo os procedimentos matemáticos, Mikhailov encontra solução desejada do problema, que é obtida como segue:

$$
\begin{aligned}
T(M, \tau)=\sum_{i=1}^{\infty} & G_{i} \psi_{i}(M) \exp \left(\int_{0}^{\tau} \frac{\beta(\tau)-\mu_{i}^{2}}{\varphi(\tau)} d \tau\right)\left\{\int_{V} w(M) \psi_{i}(M) f_{0}(M) d V\right. \\
& +\int_{0}^{\tau} \frac{1}{\varphi(\tau)} \exp \left(\int_{0}^{\tau} \frac{\mu_{i}^{2}-\beta(\tau)}{\varphi(\tau)} d \tau\right)\left[\int_{S} K(N) f(N, \tau) \frac{\psi_{i}(N)-\frac{\partial \psi_{i}(N)}{\partial n}}{A(N)+B(N)} d S\right. \\
& \left.\left.+\int_{V} \psi_{i}(N) P(M, \tau) d V\right] d \tau\right\}
\end{aligned}
$$

Após a leitura, podemos perceber que, Mikhailov ao utilizar as ideias da Transformada Integral Finita de Koshlyakov (1936), com um núcleo de transformada parecido com a sugestão de Grinberg (1948), propõe uma evolução que será de especial interesse para os pesquisadores de fenômenos difusivos. Para muitos físicos e matemáticos, encontrar essa solução aumenta sensibilidade do problema físico em questão, sendo mais fiel a realidade do experimento. Esse fato fica evidente na introdução desse artigo, e poderá ser percebido nas justificativas de Özisik e Mikhailov (1984) sobre o desenvolvimento da CITT e GITT, respectivamente.

\section{Considerações finais}

Ao pesquisarmos a técnica e realizar um levantamento histórico aprofundado, encontramos informações que relacionavam livros, artigos e datas de modo a construir uma linha do tempo bem clara. Acreditamos que após a leitura deste texto, podemos entender que a técnica proposta por Koshlyakov em 1936, foi aproveitada por diversos estudiosos como Grinberg e Mikhailov, que de certo modo ampliaram suas ideias e mostraram um novo modo de se resolver EDP que modelavam problemas clássicos da física. Com um futuro a ser descrito, com o desenvolvimento da CITT e GITT. 
Uma Construção Histórica das Técnicas da Transformada Integral Clássica (CITT) e Generalizada (GITT): Aspectos Iniciais

Reynaldo D'Alessandro Neto

\section{Referências}

COTTA, R. M. The Unified Integral Transforms (UNIT) Algorithm With Total and Partial Transformation: A Tribute to prof. Mikhail D. Mikhailov. 14th Brazilian Congress of Thermal Sciences and Engineering, Rio de Janeiro, RJ. 2012.

COTTA, R.M., KNUPP, D.C., QUARESMA, J.N.N. Analytical Methods in Heat Transfer, In: Handbook of Thermal Science and Engineering, Chapter 1, Francis A. Kulacki et al., Eds., Springer International Publishing. 2017.

D'AMBROSIO, U. A interface entre história e matemática: uma visão históricopedagógica. In: FOSSA, John A. (org.). Facetas do diamante: ensaios sobre educação matemática e história da matemática. Rio Claro: Editora da SBHMat. 2000.

DEAKIN, M. A. B. Euler's Invention of Integral Transforms. Archive for History of Exact Sciences 33 pp. 307-319. 1985.

EULER, L. Institutiones Calculi Integral. VoL 2 (Book 1, Part 2, Section 1). St. Petersburg: Imp. Acad. Sci. Reprinted as Op. Omn. I 12. 1769

EULER, L. Constructio aequationis differentio-differentialis sumto elemento du constante. Novi Comm. Acad. Sei. Petrop. 8, 150-156. Op. Omn. I 22. 395-402. 1763.

FOURIER, J.B. (1822) Théorie Analytique de la Chaleur. Firmin Didot Père \& Fils, Paris. Transl. "The analytical theory of heat (unabridged)". Cosimo, New York. 2007.

FOURIER, J.B. Théorie de la Propagation de la Chaleur dans les solides, manuscript., Bibliothèque de l'Êcole des Ponts et Chassées, Paris. 1807.

GRINBERG, G.A. Selected Problems of Mathematical Theory of Electrical and Magnetic Effects (in russian). Akad: Nauk SSSR. 1948.

KOSHLYAKOV, N.S. Basic Differential Equations of Mathematical Physics. Moscow. 1936.

KOSHLYAKOV, N.S.; SMIRNOV, M.M.; GLINER, E.B. Differential Equations of Mathematical Physics. Translated by Scripta Techinca, Inc. North-Holand Publishing Company, New York. 1964.

LUIKOV, A.V. Heat and Mass Transfer. Mir Publishers. Moscow. 1972.

MIKHAILOV, M.D. General Solution of the Heat Equation in Finite Region. International Journal Engineering Sciences, vol. 7, pp. 577-591. 1972.

ÖZISIK, M.N.; MIKHAILOV, M.D. Unified Analysis and Solutions of Heat and Mass Diffusion. John Wiley, New York. 1984. 
Uma Construção Histórica das Técnicas da Transformada Integral Clássica (CITT) e Generalizada

(GITT): Aspectos Iniciais

Reynaldo D'Alessandro Neto

ÖZISIK, M.N.; MURRAY, R.L. On the solution of linear diffusion problems with variable boundary condition parameters. Journal of Heat Transfer, 96c:48-51. 1974.

SNEDDON, I. M. The Use of Integral Transforms. New York: McGraw-Hill. 1972. TRANTER, C.J. Integral Transform in Mathematical Physics. New York: John Wiley. 1962. 